\title{
Localized large sums of random variables
}

\author{
Kevin Ford ${ }^{1, *}$ \\ Department of Mathematics, University of Illinois at Urbana-Champaign, \\ 1409 West Green St., Urbana, IL, 61801, USA \\ Gérald Tenenbaum \\ Institut Élie Cartan, Université Henri-Poincaré Nancy 1, \\ B.P. 239, 54506 Vandouvre-lès-Nancy Cedex, France
}

\begin{abstract}
We study large partial sums, localized with respect to the sums of variances, of a sequence of centered random variables. An application is given to the distribution of prime factors of typical integers.
\end{abstract}

Key words: sums of random variables, prime divisors 1991 MSC: 60G50, 11N25

Dedicated to the memory of Walter Philipp

\section{Introduction}

Consider random variables $X_{1}, X_{2}, \ldots$ with $\mathbb{E} X_{j}=0$ and $\mathbb{E} X_{j}^{2}=\sigma_{j}^{2}$. Let

$$
S_{n}=X_{1}+\cdots+X_{n}, \quad s_{n}^{2}=\sigma_{1}^{2}+\cdots+\sigma_{n}^{2},
$$

and assume that (a) $s_{n} \rightarrow \infty$ as $n \rightarrow \infty$.

* Corresponding author.

Email addresses: fordemath . uiuc . edu (Kevin Ford), gerald.tenenbaum@iecn.u-nancy. fr (Gérald Tenenbaum).

1 Research supported by National Science Foundation Grants DMS-0301083 and DMS0555367 
Given a positive function $f_{N} \geqslant 1+1 / N$, we are interested in the behavior of

$$
I=\liminf _{N \rightarrow \infty} \max _{N<s_{n}^{2} \leqslant N f_{N}}\left|S_{n}\right| / s_{n}
$$

If we replace lim inf by lim sup, it immediately follows from the law of the iterated logarithm that $I=\infty$ almost surely when $f_{N}$ is bounded. Our results answer a question originally raised, in oral form, by A. Sárközy and for which a partial answer had previously been given by the second author, see Chap. 3 of Oon (2005).

\section{Independent random variables}

Assume that the $X_{j}$ are independent. Then $\mathbb{E} S_{n}^{2}=s_{n}^{2}$. In addition to condition (a), we will work with two other mild assumptions, (b) $s_{j+1} / s_{j} \ll 1$ when $s_{j}>0$ and (c) for every $\lambda>0$, there is a constant $c_{\lambda}>0$ such that if $n$ is large enough and $s_{m}^{2}>2 s_{n}^{2}$, then

$$
\mathbb{P}\left(\left|S_{m}-S_{n}\right| \geqslant \lambda s_{m}\right) \geqslant c_{\lambda}
$$

Condition (b) says that no term in $S_{n}$ dominates the others. Condition (c) follows if the Central Limit Theorem (CLT) holds for the sequence of $S_{n}$, since CLT for $S_{n}$ implies CLT for $S_{m}-S_{n}$ as $(m-n) \rightarrow \infty$. For example, (c) holds for i.i.d. random variables, under the Lindeberg condition

$$
\forall \varepsilon>0, \quad \lim _{n \rightarrow \infty} \sum_{1 \leqslant j \leqslant n} \mathbb{E}\left(X_{j}^{2} / s_{n}^{2}:\left|X_{j}\right|>\varepsilon s_{n}\right)=0
$$

and the stronger Lyapunov condition

$$
\exists \delta>0: \sum_{1 \leqslant j \leqslant n} \mathbb{E}\left|X_{j}\right|^{2+\delta}=o\left(s_{n}^{2+\delta}\right) .
$$

Condition (c) is weaker, however, than CLT.

Theorem 1 (i) Suppose $(a),(b)$, and $f_{N}=(\log N)^{M}$ for some constant $M>0$. Then $I<\infty$ almost surely.

(ii) Suppose (a), (b), (c) and $f_{N}=(\log N)^{\xi(N)}$ with $\xi(N)$ tending monotonically to $\infty$. Then $I=\infty$ almost surely.

Remark. In the first statement of the theorem we show in fact that almost surely $I \leqslant 15 \sqrt{M+1}\left(\max _{s_{j}>0} s_{j+1} / s_{j}\right)^{2}$.

Lemma 2 (Kolmogorov's inequality, 1929) We have

$$
\mathbb{P}\left(\max _{1 \leqslant j \leqslant k}\left|S_{j}\right| \geqslant \lambda s_{k}\right) \leqslant 1 / \lambda^{2} \quad(k \geqslant 1) .
$$


Proof of Theorem 1. By (a) and (b), there is a constant $D$ so that $s_{j+1} / s_{j} \leqslant D$ for all large $j$. Define

$$
h(n):=\max \left\{k: s_{k}^{2} \leqslant n\right\} \quad\left(n \in \mathbb{N}^{*}\right),
$$

so that the conditions $N<s_{n}^{2} \leqslant N f_{N}$ and $h(N)<n \leqslant h\left(N f_{N}\right)$ are equivalent.

We first consider the case when $f_{N}:=(\log N)^{M}$. Let

$$
N_{j}:=j^{(M+3) j}, \quad t(j):=\lfloor(M+1)(\log j) / \log 2\rfloor, \quad H_{j}:=2^{t(j)},
$$

and

$$
U_{j}:=h\left(N_{j}\right), \quad U_{j, t}:=h\left(2^{t} N_{j}\right) \quad(0 \leqslant t \leqslant t(j)), \quad V_{j}:=h\left(H_{j} N_{j}\right)=U_{j, t(j)} .
$$

It is possible that $U_{j, t+1}=U_{j, t}$ for some $t$. Note that for large $j, H_{j} N_{j} \geqslant N_{j} f_{N_{j}}$.

Let $k$ be a constant depending only on $M$ and $D$. For $j \geqslant 1$ define the events

$$
\begin{aligned}
A_{j} & :=\left\{\left|S_{V_{j}}\right| \leqslant s_{U_{j+1}}\right\}, \\
B_{j} & :=\bigcap_{0 \leqslant t \leqslant t(j)-1} B_{j, t} \text { where } B_{j, t}:=\left\{\max _{U_{j+1, t} \leqslant n \leqslant U_{j+1, t+1}}\left|S_{U_{j+1, t+1}}-S_{n}\right| \leqslant k s_{U_{j+1, t}}\right\}, \\
C_{j} & :=\left\{\left|S_{U_{j+1}}-S_{V_{j}}\right| \leqslant 2 s_{U_{j+1}}\right\} .
\end{aligned}
$$

By (b) and the definition of $h(N)$, we have

$$
D^{-1} \sqrt{2^{t} N_{j}} \leqslant s_{U_{j, t}} \leqslant \sqrt{2^{t} N_{j}}
$$

for all $j, t$. It follows from Lemma 2 that

$$
\mathbb{P}\left(\overline{A_{j}}\right) \leqslant D^{2} \frac{H_{j} N_{j}}{N_{j+1}} \leqslant \frac{D^{2}}{j^{2}} .
$$

Thus, $\sum_{j \geqslant 1} \mathbb{P}\left(\overline{A_{j}}\right)<\infty$ and hence almost surely there is a $j_{0}$ so that $A_{j}$ occurs for $j \geqslant j_{0}$. Applying Lemma 2 again yields

$$
\mathbb{P}\left(\overline{B_{j, t}}\right) \leqslant \frac{s_{U_{j+1, t+1}}^{2}-s_{U_{j+1, t}}^{2}}{k^{2} s_{U_{j+1, t}}^{2}} \leqslant \frac{D^{2} 2^{t+1} N_{j+1}}{k^{2} 2^{t} N_{j+1}}=\frac{2 D^{2}}{k^{2}} .
$$

If $k=3 D \sqrt{M+1}$, then

$$
\mathbb{P}\left(B_{j}\right) \geqslant\left(1-\frac{2 D^{2}}{k^{2}}\right)^{t(j)} \geqslant \frac{1}{j^{1 / 2}}
$$

for large $j$. Also by Lemma $2, \mathbb{P}\left(C_{j}\right) \geqslant \frac{3}{4}$, and since $B_{j}$ and $C_{j}$ are independent,

$$
\sum_{j \geqslant 1} \mathbb{P}\left(B_{j} C_{j}\right)=\infty
$$


Since the events $B_{j} C_{j}$ are independent, the Borel-Cantelli lemma implies that almost surely the events $B_{j} C_{j}$ occur infinitely often. Thus, the event $A_{j} B_{j} C_{j}$ occurs for an infinite sequence of integers $j$. Take such a index $j$, let $n \in\left[U_{j+1}, V_{j+1}\right]$ and $U_{j+1, g-1}<n \leqslant U_{j+1, g}$, where $1 \leqslant g \leqslant t(j+1)$. We have by several applications of (1)

$$
\begin{aligned}
\left|S_{n}\right| & \leqslant\left|S_{V_{j}}\right|+\left|S_{U_{j+1}}-S_{V_{j}}\right|+\sum_{0 \leqslant t \leqslant g-2}\left|S_{U_{j+1, t}}-S_{U_{j+1, t+1}}\right|+\left|S_{n}-S_{U_{j+1, g-1}}\right| \\
& \leqslant 3 s_{U_{j+1}}+k \sum_{0 \leqslant t \leqslant g-1} s_{U_{j+1, t}} \\
& \leqslant\left\{3+k\left(1+2^{1 / 2}+\cdots+2^{(g-1) / 2}\right)\right\} \sqrt{N_{j+1}} \\
& \leqslant 5 k \sqrt{2^{g-1} N_{j+1}} \\
& \leqslant 5 k D s_{n}=15 D^{2}(M+1)^{1 / 2} s_{n} .
\end{aligned}
$$

This completes the proof of part (i) of the theorem, since

$$
V_{j+1} \geqslant h\left(\frac{1}{2} j^{M+1} N_{j}\right) \geqslant h\left(N_{j} \log ^{M} N_{j}\right)
$$

for large $j$.

Now suppose $f_{N}=(\log N)^{\xi(N)}$ with $\xi(N)$ tending monotonically to $\infty$.

Let $\lambda>0$ be arbitrary and define $K:=2 D^{2}$. Let $N_{1}^{*}$ be so large that $f_{N_{1}^{*}} \geqslant K$. For $j \geqslant 1$ let $N_{j+1}^{*}=N_{j}^{*} K^{u(j)}$, where $u(j):=\left\lfloor\log f_{N_{j}^{*}} / \log K\right\rfloor$. Put

$$
U_{j}^{*}:=h\left(N_{j}^{*}\right), \quad U_{j, t}^{*}:=h\left(K^{t} N_{j}^{*}\right)(0 \leqslant t \leqslant u(j)) .
$$

Let $J_{j}:=\left[U_{j}^{*}, U_{j+1}^{*}\right]$ and

$$
Y_{j}:=\max _{n \in J_{j}}\left|S_{n}\right| / s_{n}
$$

We have

$$
u(j) \geqslant 1 \Rightarrow N_{j+1}^{*} \geqslant K N_{j}^{*} \Rightarrow u(j) / \log j \rightarrow \infty .
$$

Therefore, by (c), if $j$ is sufficiently large then

$$
\begin{aligned}
\mathbb{P}\left(Y_{j} \leqslant \lambda / 2\right) & \leqslant \prod_{1 \leqslant t \leqslant u(j)} \mathbb{P}\left(\left|S_{U_{j, t}^{*}}-S_{U_{j, t-1}^{*}}\right| \leqslant \frac{1}{2} \lambda\left(s_{U_{j, t}^{*}}+s_{U_{j, t-1}^{*}}\right)\right) \\
& \leqslant \prod_{1 \leqslant t \leqslant u(j)} \mathbb{P}\left(\left|S_{U_{j, t}^{*}}-S_{U_{j, t-1}^{*}}\right| \leqslant \lambda \sqrt{K^{t} N_{j}^{*}}\right) \\
& \leqslant\left(1-c_{\lambda}\right)^{u(j)} \leqslant \frac{1}{j^{2}} .
\end{aligned}
$$

Thus

$$
\sum_{j \geqslant 1} \mathbb{P}\left(Y_{j} \leqslant \lambda / 2\right)<\infty .
$$

Almost surely, $Y_{k} \leqslant \lambda / 2$ for only finitely many $k$. 
Theorem 1 has an analog for Brownian motion, which follows from Theorem 1 and the invariance principle.

Theorem 3 Let $W(t)$ be Brownian motion on $[0, \infty)$. If $f_{N}=(\log N)^{M}$ with fixed $M>0$, then almost surely

$$
I=\liminf _{N \rightarrow \infty} \max _{N<t \leqslant N f_{N}} \frac{|W(t)|}{\sqrt{t}}<\infty .
$$

If $f_{N}=(\log N)^{\xi(N)}$ with $\xi(N) \rightarrow \infty$, then $I=\infty$ almost surely.

Theorem 3 can be proved directly and more swiftly using the methods used to establish Theorem 1. By invariance principles (e.g. Philipp, 1986), one may deduce from Theorem 3 a version of Theorem 1 where stronger hypotheses on the $X_{j}$ are assumed. As it stands, now, however, Theorem 1 does not follow from Theorem 3.

\section{Dependent random variables}

The conclusions of Theorem 1 can also be shown to hold for certain sequences of weakly dependent random variables by making use of almost sure invariance principles. We assume that (d) there exists a sequence of i.i.d. normal random variables $Y_{j}$ with $\mathbb{E} Y_{j}^{2}=\sigma_{j}^{2}$, defined on the same probability space as the sequence of $X_{j}$, and such that if $Z_{n}=Y_{1}+\cdots+Y_{n}$, then

$$
\left|S_{n}-Z_{n}\right|=O\left(s_{n}\right) \quad \text { a.s. }
$$

Of course the variables $Y_{j}$ are dependent on the $X_{j}$, but not on each other. Property (d) has been proved for martingale difference sequences, sequences satisfying certain mixing conditions, and lacunary sequences $X_{j}=\left\{n_{j} \omega\right\}$ with inf $n_{j+1} / n_{j}>1$, $\omega$ uniformly distributed in $[0,1]$ and $\{x\}$ is the fractional part of $x$. See e.g. Philipp (1986) for a survey of such results.

Theorem 4 (i) Suppose $(a),(b)$, and $(d)$. If $f_{N}:=(\log N)^{M}$ for some constant $M>0$, then $I<\infty$ almost surely.

(ii) Let $\xi(N)$ tend monotonically to $\infty$ and set $f_{N}:=(\log N)^{\xi(N)}$. Then $I=\infty$ almost surely.

By (d),

$$
I=O(1)+\liminf _{N \rightarrow \infty} \max _{N<s_{n}^{2} \leqslant N f_{N}}\left|Z_{n}\right| / s_{n},
$$

and we apply Theorem 1 to the sequence of $Y_{j}$. The variable $Z_{n}$ is normal with variance $s_{n}^{2}$, hence (c) holds. 


\section{Prime factors of typical integers}

Consider a sequence of independent random variables $Y_{p}$, indexed by prime numbers $p$, such that $\mathbb{P}\left(Y_{p}=1\right)=1 / p$ and $\mathbb{P}\left(Y_{p}=0\right)=1-1 / p$. We can think of $Y_{p}$ as modelling whether or not a "random" integer is divisible by $p$. As $\mathbb{E} Y_{p}=1 / p$, we form the centered r.v.'s $X_{p}=Y_{p}-1 / p$ (we may also define $X_{j}$ for non-prime $j$ to be zero with probability 1 ). Let

$$
T_{n}=\sum_{p \leqslant n} Y_{p}, \quad S_{n}=\sum_{p \leqslant n} X_{p} .
$$

We have $\mathbb{E} X_{p}^{2}=(1-1 / p) / p$, hence by Mertens' estimate

$$
s_{n}^{2}=\sum_{p \leqslant n} \frac{1}{p}-\frac{1}{p^{2}}=\log _{2} n+O(1) .
$$

Here and in the sequel, $\log _{k}$ denotes, for integer $k \geqslant 2$, the $k$-fold iterated logarithm. Since $\mathbb{E}\left|X_{p}\right|^{3} \leqslant 1 / p$, the Lyapunov condition holds with $\delta=1$. Then (a), (b) and (c) hold, and therefore the conclusion of Theorem 1 holds. Here take $D=\max _{n \geqslant 2} s_{n+1} / s_{n}$ since $s_{1}=0$.

Let $\omega(m, t)$ denote the number of distinct prime factors of $m$ which are $\leqslant t$. The sequence $\left\{T_{n}: n \geqslant 1\right\}$ mimics well the behavior of the function $\omega(m, n)$ for a "random" $m$, at least when $n$ is not too close to $m$. This is known as the Kubilius model. It can be made very precise, see (Elliott , 1979, Ch. 3, especially pp. 119122) and Tenenbaum (1999) for the sharpest estimate known to date. Suppose $r$ is an integer with $2 \leqslant r \leqslant x$ and $r=x^{1 / u}, \boldsymbol{\omega}_{r}(m)=(\omega(m, 1), \ldots, \omega(m, r))$ and suppose $Q$ is any subset of $\mathbb{Z}^{r}$. Then, given arbitrary $c<1$, and uniformly in $x, r$ and $Q$, we have

$$
\frac{1}{x}\left|\left\{m \leqslant x: \omega_{r}(m) \in Q\right\}\right|=\mathbb{P}\left(\left(T_{1}, \ldots, T_{r}\right) \in Q\right)+O\left(x^{-c}+\mathrm{e}^{-u \log u}\right) .
$$

An analog of Theorem 1, established by parallel estimates, provides via (2) information about localized large values of

$$
\varrho(m, t):=\left|\omega(m, t)-\log _{2} t\right| / \sqrt{\log _{2} t} .
$$

Theorem 5 (i) Let $M>0$ be fixed, $f_{N}:=(\log N)^{M}$ and put $K:=30 D^{2} \sqrt{M+1}$. If $g=g(m) \rightarrow \infty$ monotonically as $m \rightarrow \infty$ in such a way that $g^{2} f_{g^{2}} \leqslant \log _{2} m$ for large $m$, then for a set of integers $m$ of natural density $1,{ }^{2}$ we have

$$
\min _{g(m) \leqslant N \leqslant g(m)^{2}} \max _{N<\log _{2} t \leqslant N f_{N}} \varrho(m, t) \leqslant K
$$

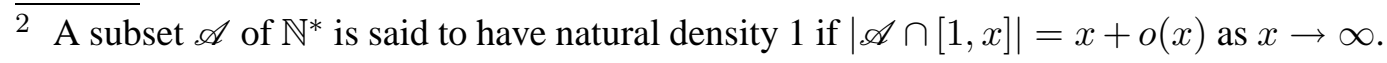


(ii) Let $\xi(N) \rightarrow \infty$ in such a way that $f_{N}:=(\log N)^{\xi(N)} \leqslant N$. Suppose that $g(m) \rightarrow \infty$ monotonically as $m \rightarrow \infty$, that $g(m) \leqslant\left(\log _{2} m\right)^{1 / 10}$, and let

$$
I_{m}:=\min _{\substack{g(m) \leqslant N \\ N f_{N} \leqslant \log _{2} m}} \max _{N \leqslant \log _{2} t \leqslant N f_{N}} \varrho(m, t) .
$$

Then, $I_{m} \rightarrow \infty$ on a set of integers $m$ of natural density 1 .

We follow the proof of Theorem 1. Keeping the notation introduced there, we see that for large $J$,

$$
\mathbb{P}\left(\bigcap_{J \leqslant j \leqslant 3 J / 2} \overline{A_{j} B_{j} C_{j}}\right) \leqslant \sum_{J \leqslant j \leqslant 3 J / 2} \frac{D^{2}}{j^{2}}+\prod_{J \leqslant j \leqslant 3 J / 2}\left(1-\frac{3}{4 \sqrt{j}}\right) \ll \frac{1}{J} .
$$

For large $G$, define $J$ by $N_{J+1}<G \leqslant N_{J+2}$. Then $G^{5 / 3}>N_{\lfloor 3 J / 2\rfloor+2}$ and $J \gg_{M}$ $(\log G) / \log _{2} G$. Thus, for large $G$,

$$
\mathbb{P}\left(\min _{G \leqslant N \leqslant G^{5 / 3}} \max _{h(N)<n \leqslant h\left(N f_{N}\right)} \frac{\left|S_{n}\right|}{s_{n}} \leqslant K\right) \geqslant 1-O\left(\frac{1}{J}\right) \geqslant 1-O\left(\frac{\log _{2} G}{\log G}\right) .
$$

The direct number theoretic analog of $\left|S_{n}\right| / s_{n}$ is

$$
\widetilde{\varrho}(m, t):=\frac{\left|\omega(m, t)-\sum_{p \leqslant t} 1 / p\right|}{\sqrt{\sum_{p \leqslant t}(1-1 / p) / p}} .
$$

By (2), if $G$ is large and $G \leqslant \sqrt{\log _{2} x}$ (so that $G^{5 / 3} f_{G^{5 / 3}} \leqslant\left(\log _{2} x\right)^{7 / 8}$ ), then

$$
\frac{1}{x}\left|\left\{m \leqslant x: \min _{G \leqslant N \leqslant G^{5 / 3}} \max _{h(N)<n \leqslant h\left(N f_{N}\right)} \widetilde{\varrho}(m, t) \leqslant K\right\}\right| \geqslant 1-O\left(\frac{\log _{2} G}{\log G}\right) .
$$

Since $\widetilde{\varrho}(m, t)=\varrho(m, t)+O\left(1 / \sqrt{\log _{2} t}\right)$, the first part of the theorem follows.

The second part is similar. Note that $\omega(n, x)-\omega\left(n, x^{1 / \sqrt{\log _{2} x}}\right) \leqslant \sqrt{\log _{2} x}$ for $n \leqslant x$, and, for brevity, write $g=g(\sqrt{x})$. By (2) with $u:=\sqrt{\log _{2} x}$, we have, for any fixed $K$ and large $x$,

$$
\begin{aligned}
& \frac{1}{x}\left|\left\{m \leqslant x: \min _{\substack{N \geqslant g \\
N f_{N} \leqslant \log _{2} m}} \max _{N<\log _{2} t \leqslant N f_{N}} \widetilde{\varrho}(m, t) \leqslant K\right\}\right| \\
& \leqslant \frac{1}{x}\left|\left\{\sqrt{x} \leqslant m \leqslant x: \min _{\substack{N \geqslant g \\
N f_{N} \leqslant \mathscr{L}(x)}} \max _{N \leqslant \log _{2} t \leqslant N f_{N}} \widetilde{\varrho}(t) \leqslant K+2\right\}\right|+\frac{1}{\sqrt{x}} \\
& \leqslant \mathbb{P}\left(\inf _{\substack{N \geqslant g \\
N f_{N} \leqslant \mathscr{L}(x)}} \max _{h(N)<n \leqslant h\left(N f_{N}\right)} \frac{\left|S_{n}\right|}{s_{n}} \leqslant K+2\right)+O\left(\frac{1}{\log _{2} x}\right),
\end{aligned}
$$


where $\mathscr{L}(x):=\log _{2} x-\frac{1}{2} \log _{3} x$. Since $f_{N} \leqslant N$, we have $N_{j+1}^{*} \leqslant\left(N_{j}^{*}\right)^{2}$ in the notation of the proof of Theorem 1. The interval

$$
\left[\left(\log _{2} x\right)^{1 / 10}, \mathscr{L}(x)^{1 / 2}\right]
$$

therefore contains at least one interval $J_{j}$. By the proof of Theorem 1 , for large $x$, the probability above does not exceed $\sum_{j \geqslant j_{0}} 1 / j^{2} \leqslant 1 /\left(j_{0}-1\right)$, where $j_{0} \rightarrow \infty$ as $x \rightarrow \infty$.

Remarks. The upper bound $g^{2}$ of $N$ in the first part can be sharpened. By the same methods, similar results can be proved for a wide class of additive arithmetic functions $r(m, t)=\sum_{p^{a} \| m} r\left(p^{a}\right)$ in place of $\omega(m, t)$.

Acknowledgment. The authors are indebted to Walter Philipp for helpful discussions on the use of almost sure invariance principles.

\section{References}

P.D.T.A. Elliott, 1979. Probabilistic Number Theory, I, Springer-Verlag, New York. S.M. Oon, 2005. Construction des suites binaires pseudo-aléatoires, Thèse d'université, Nancy, Université Henri Poincaré-Nancy 1, UFR STMIA, 110 pp.

W. Philipp, 1986. Invariance principles for independent and weakly dependent random variables, in Dependence in Probability and Statistics (Oberwolfach, 1985), 225-268, Progr. Probab. Statist. 11, Birkhäuser Boston, Boston, MA.

G. Tenenbaum, 1999. Crible d'Ératosthène et modèle de Kubilius, in: K. Gyôry, H. Iwaniec, J. Urbanowicz (eds.), Number Theory in Progress, Proceedings of the conference in honor of Andrzej Schinzel, Zakopane, Poland 1997, 1099-1129, Walter de Gruyter, Berlin, New York. 